\title{
Uterovaginal Prolapse in a Newborn with Meningomyelocele:
} \section{Case Report}

\author{
Alper Aykanat MD ${ }^{1, *}$, Ebru Solakoğlu MD ${ }^{2}$, Burçak Bilginer MD ${ }^{3}$, Hasan Tolga Çelik MD ${ }^{1}$, Şule Yiğit MD ${ }^{1}$ \\ ${ }^{1}$ Division of Neonatology, Department of Pediatrics, Hacettepe University Faculty of Medicine, Ankara, Turkey \\ ${ }^{2}$ Department of Pediatrics, Hacettepe University Faculty of Medicine, Ankara, Turkey \\ ${ }^{3}$ Department of Neurosurgery, Hacettepe University Faculty of Medicine, Ankara, Turkey
}

\section{A B S T R A C T}

Background: Neural tube defects are a group of congenital malformations in which the spinal column is bifid as a result of failed closure of the embryonic neural tube. Although not common, they might be complicated with pelvic organ prolapse mostly due to abnormal innervation and the resulting atrophy of the pelvic floor musculature.

Case: In this case report we present a newborn with uterovaginal prolapse in the setting of meningomyelocele, in whom the prolapse of pelvic organs spontaneously ameliorated after surgical correction of meningomyelocele.

Key Words: Newborn, Uterovaginal prolapse, Meningomyelocele, Neurosurgical correction

\section{Introduction}

Meningomyelocele is the most common neural tube defect (NTD). Affected individuals might experience hydrocephalus, motor deficits (weakness of the lower limbs, urinary and fecal incontinence), sensory disturbances, and orthopedic abnormalities (club foot, contractures of the lower limbs, hip dislocation, scoliosis, kyphosis)., ${ }^{1,2}$ Although uncommon, prolapse of the genital organs might also be seen in patients with severe forms of meningomyelocele, which is thought to result from the lack of innervation of the pelvic floor muscles. ${ }^{3-5}$ In this case report we present a newborn with uterovaginal prolapse associated with meningomyelocele.

\section{Case}

A baby girl weighing $3170 \mathrm{~g}$ (40th percentile) with a congenital NTD was born to a 29 -year-old mother by cesarean section at the 38th gestational week. She was admitted to the neonatal intensive care unit, and antibiotic treatment was initiated because the meningomyelocele sac had spontaneously perforated during labor.

There was parental consanguinity. The mother also had gestational diabetes controlled by diet and had begun folic acid supplementation after the sixth week of gestation. Triple test screening results were high at the 18 th week of gestation. The family refused amniocentesis and therefore no genetic counseling could be done during pregnancy. A 4$\mathrm{cm}$ meningomyelocele sac along with concomitant scoliosis was noted during the 21st week of gestation, and hydrocephalus was detected at the 33rd week of gestation.

\footnotetext{
The authors indicate no conflicts of interest.

* Address correspondence to: Alper Aykanat, MD, Department of Pediatrics, Division of Neonatology, Hacettepe University Faculty of Medicine, Ankara, Turkey; Phone +905067067713

E-mail address: alper5334@windowslive.com (A. Aykanat).
}

Medical termination of the pregnancy was offered but the parents refused.

The first examination of the baby showed general hypotonicity, a $4 \times 5 \mathrm{~cm}$ meningomyelocele sac with concomitant severe scoliosis at the lumbosacral level, an occipitofrontal circumference of $36 \mathrm{~cm}$ (80th percentile) with a normotensive anterior fontanelle, bilateral rocker bottom feet, and an absence of anal tone and deep tendon reflexes in the lower limbs. Cardiovascular, respiratory, abdominal, and genitourinary examinations were normal. Chest x-ray revealed multiple costal anomalies. Hydrocephalus and Chiari type 2 malformation were confirmed with cranial ultrasonography. Echocardiography was normal.

During the first postnatal 12-24 hours of life, a previously absent pink and fleshy protuberant mass was detected projecting out from the vagina (Fig. 1). Abdominal ultrasonography revealed uterovaginal prolapse with concomitant, inferiorly displaced urinary bladder adjacent to the prolapsed tissues, hypoplastic left kidney, and minimal dilatation in the collecting system of the right kidney. Other possible causes of interlabial masses, such as urethral prolapse, ureterocele, paraurethral cyst, vaginal polyp, and rhabdomyosarcoma, were therefore excluded. The prolapse was manually reduced by a pediatric surgeon using general anesthesia with simple digital reduction on the third day of life but then reoccurred on the fourth day of life. The patient was scheduled to be followed-up without surgical intervention unless any circulatory insufficiency of the prolapsed tissue developed. Between the 4th and 14th days of life, the uterovaginal tissue showed intermittent spontaneous reduction and reprolapse without any improvement in or recovery of the defect. Routine care was given to the prolapsed tissue as coverage with moistened sponges and intermittent reevaluation for possible circulatory disorders.

On the 13th day of life, the patient underwent surgery performed by a neurosurgeon and a plastic surgery 


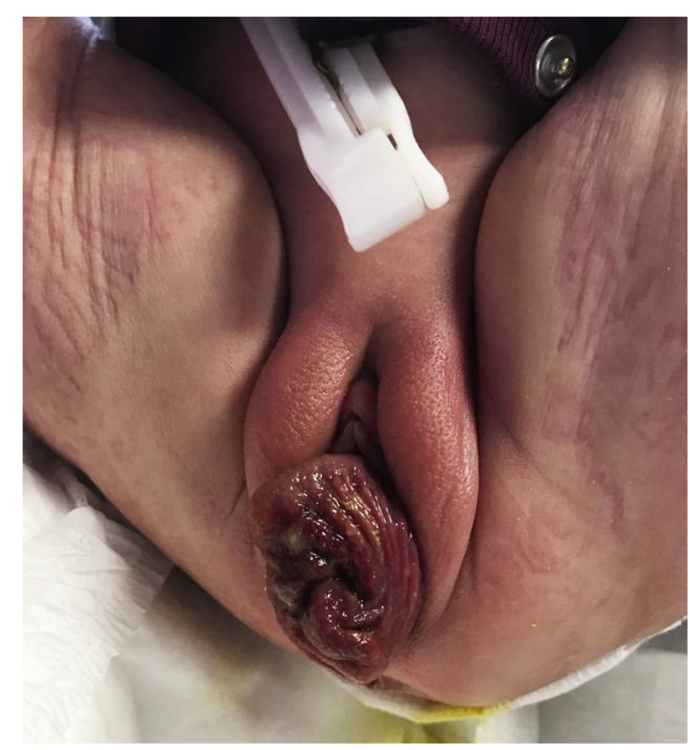

Fig. 1. Pink and fleshy protuberant mass projecting out from the vagina, which is later diagnosed as urogenital prolapse.

specialist for the NTD. To repair the large-sized dural defect, a flap shift procedure was carried out from the lumbosacral region to the midline. A ventriculoperitoneal shunt was not placed at this time. The patient was placed in a supine position for the procedure and then followed-up in the neonatal intensive care unit postoperatively. Routine care of the prolapsed uterovaginal tissue continued as was done previously. On the 17th day of life, no postoperative complications were seen regarding the repair of the meningomyelocele and the uterovaginal prolapse, which had been intermittently and consistently seen before the repair of meningomyelocele, was no longer present. Because of the neurogenic bladder and vesicourethral reflux risk, amoxicillin prophylaxis was started, and the patient was discharged on the 18th day of life to outpatient follow-up.

The patient was rehospitalized on the 25th day of life because of cerebrospinal fluid leakage from the meningomyelocele surgical scar and an accelerated increase in head circumference. A ventriculoperitoneal shunt was placed on the 31st day of life to decompress the excessive cerebrospinal fluid and lower the intracranial pressure. The patient was discharged again on the 34th day of life. During this period, the patient showed no sign of uterovaginal prolapse.

Follow-up of the patient is routinely carried out at our outpatient clinics for possible neurological, developmental, and urogenital problems. Measurements in head circumference have shown a regular course after ventriculoperitoneal shunting with no clinical evidence of increased cranial pressure. The patient was guided to physiotherapy to preserve neurological function of the lower extremities. Urine output and renal function tests were normal without a need for urinal catheterization. Voiding cystourethrography was normal at 2 months of age. Abdominal ultrasonography showed normal pelvic anatomy at 3 months of age. The patient is now 4 months of age and remains without any sign of uterovaginal prolapse.

\section{Summary and Conclusion}

NTDs are a group of congenital malformations in which the spinal column is bifid as a result of failed closure of the embryonic neural tube during the fourth week after fertilization. Myelomeningocele is the most common and most severe form, in which the spinal cord is open dorsally and forms a meningeal sac in the lumbosacral region. Affected individuals might experience hydrocephalus, motor deficits, sensory disturbances, orthopedic anomalies, and urogenital abnormalities. Surgical interventions and lifetime follow-up are needed for most of these patients. ${ }^{1,2}$

Although usually a condition seen in multiparous and postmenopausal women, uterovaginal prolapse might also be noted in newborn babies, although the pathophysiology is not yet well understood. Since the first observance in 1723 , more than 60 cases of neonatal uterovaginal prolapse have been reported in the literature and have varied in severity. These cases are divided into 2 subgroups: those with accompanying NTDs (75\% are comorbid with meningomyelocele) and those that are neuroanatomically normal $(75 \%$ with a breech presentation at labor, $25 \%$ with other etiologies, such as birth trauma, pelvic skeletal deformities, increased fetal intra-abdominal pressure, cutis laxa). It is thought that abnormal innervation and the resulting atrophy of the pelvic floor musculature might be the cause of uterovaginal prolapse in newborns with severe forms of meningomyelocele, whereas others who are neuroanatomically normal might have other undefined etiologies., 3

Conservative or surgical interventions must be undertaken as soon as possible to prevent possible vaginal injury and to help protect fertility in newborns with uterovaginal prolapse. ${ }^{4,5}$ According to the literature, several techniques may be carried out in reduction of prolapsed pelvic organs, such as conservative methods including simple digital reduction, the use of hypertonic saline packs and pessaries, or urogenital surgical methods including labial or vaginal fusion with suturing and abdominal sacrocolpopexy if the mass is not reducible, or even hysterectomy. ${ }^{3-6}$ In neuroanatomically normal newborns with uterovaginal prolapse, simple digital reduction is generally sufficient, whereas those with meningomyelocele have high recurrence rates of uterovaginal prolapse even when they are treated with urogenital surgical methods. ${ }^{3}$ Because the abnormal innervation of the pelvic floor musculature due to the NTD results in poor pelvic muscle tone and subsequent prolapse of pelvic organs, these patients benefit from surgical repair of the meningomyelocele, which improves the tone of the musculus levator ani. ${ }^{3}$

On the basis of the past experiences described in the literature, coexistence of meningomyelocele and uterovaginal prolapse in our patient in the setting of ineffective digital reduction and subsequent reprolapse without any improvement have led us to correction of the NTD in advance and to subsequent further planning of urogenital procedures if necessary.

In conclusion, management of neonatal genital prolapse differs in terms of presence or absence of concurrent NTD. Surgical correction of meningomyelocele and follow-up of expectant self-recovery of prolapse seems to be the most 
beneficial and definitive treatment in these patients. Urgent and definitive treatment of urogenital prolapse is required at all forms of the disease, to prevent possible vaginal injury and to help protect fertility.

\section{References}

1. Mutlu M, Aslan Y: Nöral Tüp Defektleri. In: Yurdakök M, editor. Yurdakök Pediyatri Kısım 6: Yenidoğan Hastalıkları. Ankara, Güneş Tıp Kitapevleri, 2018, pp 445-450
2. Copp AJ, Adzick NS, Chitty LS, et al: Spina bifida. Nat Rev Dis Primers 2015; 1: 15007

3. Henn EW, Juul L, Rensburg KV: Pelvic organ prolapse in the neonate: report of two cases and review of the literature. Int Urogynecol J 2015; 26:613

4. Lockwood G, Durkee C, Groth T: Genital prolapse causing urinary obstruction and hydronephrosis in a neonate: a case and review of the literature. J Neonat Surg 2012; 1:39

5. Hyginus EO, John CO: Congenital uterovaginal prolapse present at birth. J Surg Tech Case Rep 2013; 5:89

6. Pirgon O, Atabek ME, Suleymanoglu S: Genital prolapse in a newborn following resection of sacrococcygeal teratoma. J Pediatr Adolesc Gynecol 2009; 22:e96 